\title{
PRÁCTICAS ALIMENTARIAS Y OBESIDAD INFANTIL. CULTURA REGIONAL Y FACTORES INTRAPERSONALES FAMILIARES Y ESCOLARES
}

\author{
Food practices and childhood obesity. Regional culture and intrapersonal, \\ familiar and scholar factors
}

Martha Leticia Cabello Garza ${ }^{1}$, Silvia VÁzQueZ-GonZÁlez²

Resumen

El objetivo de este trabajo es presentar a partir de la perspectiva de los niños y las niñas que presentan obesidad, algunas categorías identificadas de los contextos intrapersonales, familiares y escolares que están articulados con las prácticas de alimentación, el aumento de peso y algunos problemas que obstaculizan estilos de vida saludable, así como de los contextos socioculturales y agentes implicados en dichas prácticas. El diseño del estudio es cualitativo, con muestreo intensional. Se aplicaron 12 entrevistas semiestructuradas a niños y niñas que cursaban el $4 .^{\circ}$ y $5 .^{\circ}$ año de primaria, en escuelas localizadas en un medio urbano del área metropolitana de Monterrey, que presentaban obesidad infantil. Se encontró que diversos factores de los contextos de desarrollo de los niños y niñas han modificado significativamente los patrones alimentarios y de ejercicio físico, entre los que destacan las condiciones socioeconómicas, los problemas de organización de la familia, distribución de tareas e implicación de los padres como cuidadores primarios, aspectos socioculturales y la influencia de los medios de información que unidas a las condiciones de la vida moderna conducen a favorecer el consumo de alimentos industrializados y de alta densidad calórica. Los niños y niñas que viven con obesidad luchan contra un estigma que afecta su autoestima y que a la vez los excluye de algunas actividades y procesos de socialización en los entornos familiar, escolar y social. Es por ello importante que se formulen políticas sociales y modelos de intervención que contribuyan a estilos de vida saludables, y en este campo la labor del trabajador social puede ser muy importante.

Palabras clave: Trabajo social y salud, obesidad infantil, patrones alimentarios regionales, prácticas alimentarias, necesidades de intervención en salud.

\begin{abstract}
The objective of this paper is to present, from the perspective of obese boys and girls, some categories identified in intrapersonal, family and school contexts which are related to feeding practices, weight gain and some of the problems that make it difficult to have healthy lifestyles. In addition, the socio-cultural contexts and actors involved in such practices are analyzed. This qualitative study was designed with intentional sampling, 12 semi-structured interviews were undertaken with boys and girls enrolled in 4th and 5 th grade who were obese, in an urban environment located in the metropolitan area of Monterrey. It was found that several factors of developing contexts of boys and girls have had a significant impact on their eating and exercise patterns. Some of them are socio- economic conditions, problems in family organization, task distribution and the engagement of parents as primary caregivers, socio-cultural factors and the influence of mass media, which combined with the conditions of modern life promote consumption of processed foods of high caloric density. Discourses show a stigma that affects their self-esteem and at the same time exclude them from some activities and processes of socialization in the family, school and social environments. It is therefore important that social policies and intervention models that contribute to healthy lifestyles are formulated, and in this field the effort of the social worker can be very important.
\end{abstract}

Keywords: Social work and health, childhood obesity, regional eating patterns, eating habits, health intervention needs

Recibido: 27/09/2014 Aceptado: 31/10/2014

Publicado: 01/12/2014

1. Facultad de Trabajo Social y Desarrollo Humano. Universidad Autónoma de Nuevo León. México. Cd Universitaria, San Nicolás de los Garza N.L. CP 66451 Tel (52) 8183 521309. E-mail: marthacabello@hotmail.com

2. Universidad Autónoma de Tamaulipas. México. 


\section{Introducción}

La obesidad infantil es actualmente para México y el mundo, un problema de salud pública prioritario, debido a las altas tasas de prevalencia que se han presentado en las últimas décadas. Según la última Encuesta Nacional de Salud y Nutrición en México (ENSANUT, 2012), 22 millones de mexicanos presentan obesidad y más de una tercera parte de la población (el 34.4\%) entre 5 y 11 años de edad, presenta exceso de peso corporal y aunque se señala que disminuyó ligeramente su incidencia ( $1.1 \%$ menos en comparación con la ENSANUT del 2006), las cifras aún son alarmantes, además, la misma fuente señala que el $23 \%$ de la ingesta diaria de alimentos proviene de alimentos altos en azúcares y grasas, lo cual refleja una alimentación basada en alimentos de alta densidad energética y pobre en nutrientes, esto plantea un gran reto a los sistemas de salud.

En el Estado de Nuevo León en México, según datos del 2012, la prevalencia de inseguridad alimentaria en los hogares fue de $62.6 \%$ de los cuales $37.8 \%$ mostraba inseguridad leve, $17.8 \%$ inseguridad moderada y $7 \%$ inseguridad severa, con mayor prevalencia en el medio rural. Con relación al sobrepeso y obesidad en los niños y niñas en edad escolar, la prevalencia fue de $40.2 \%$ siendo $20.4 \%$ de sobrepeso y $19.8 \%$ de obesidad, con cifras mayores para los niños (43.9\%) que para las niñas (36.4). Del año 2006 al 2012 la prevalencia aumentó en comunidades rurales y disminuyó en las urbanas (ENSANUT, Nuevo León, 2012).

La dieta tradicional constituida por alimentos frescos del mercado regional, paulatinamente han sido sustituidos por productos industrializados, lo que es favorecido por la incorporación de la mujer al mercado de trabajo, los problemas de organización y la inequitativa distribución de tareas en el hogar. Por otra parte, la reducción del tiempo destinado a la preparación de la comida sana en casa, el auge de la comida de compra rápida, la movilidad pasiva de las personas en los medios de transporte predominantes, la escasa práctica del deporte y las actividades de ocio sedentarias, son factores que cada vez más alejan a las personas de lo que es considerado como «estilo de vida saludable».

Los efectos de las condiciones señaladas se manifiestan en un peso corporal alejado de los estándares de la salud y de la normalidad para una edad determinada, lo que no sólo se expresa como un problema biológico, sino en un problema social susceptible de ser analizado en un conjunto de dimensiones que son objeto de atención por las y los trabajadores sociales, ya que la salud es un derecho básico de la ciudadanía. No hay bienestar y desarrollo sin salud y un imperativo de la profesión es dar respuesta a las nuevas condiciones 
sociales, uno de ellos las alarmantes cifras de obesidad en todos los grupos de edad, de los cuales este estudio se concentra en el de los niños en edad escolar.

Este estudio retoma algunos fundamentos de la teoría ecológica y del interaccionismo simbólico. La teoría ecológica de Bronfenbrenner (1979), se centra en el estudio de las personas en sus ambientes naturales (la escuela, el barrio, la casa donde viven, etc.). Esta teoría concibe al ambiente ecológico como un conjunto de estructuras clasificadas y ordenadas en diferentes niveles, en donde cada uno contiene al otro. Bronfenbrenner denomina a esos niveles el microsistema, el mesosistema, el exosistema y el macrosistema. Para este estudio se considera el microsistema como el nivel más inmediato en el que se desarrolla el niño que es el nivel intrapersonal; el mesosistema comprende las interrelaciones de dos o más entornos en los que el niño participa activamente, como lo es el ambiente familiar y escolar; y finalmente, el macrosistema que lo configuran la cultura y la subcultura en la que se desenvuelve el niño. Al respecto, Saltier (2004) menciona que el modelo ecológico está dirigido a los cambios del entorno, conductas y normas que ayudan a que las personas tomen decisiones saludables en su vida cotidiana. El fundamento del modelo ecológico parte del concepto de que la conducta no se cambia en aislamiento y que es necesario un entorno de apoyo para que las personas tomen decisiones saludables, lo que proporciona un encuadre a partir del cual se puede entender cómo es que el entorno influye y afecta la percepción de cada persona hacia su bienestar y desarrollo armónico (Payne, 1995).

Para Villalba (2004, p. 288) las perspectivas ecológicas ponen el «énfasis en la interacción entre sistemas y contextos y en identificar recursos y fortalezas... incluyen el mundo interno de las personas y la identificación de sus capacidades de autocuidado, autodirección, autoeficacia, autocontrol y autoestima».

El interaccionismo simbólico es una de las orientaciones metodológicas que comparten las ideas básicas del proceso hermenéutico o interpretativo. De acuerdo a Pérez (2004), en el abordaje hermenéutico, la realidad está constituida no sólo por hechos observables y externos, sino también por significados, símbolos e interpretaciones elaboradas por el propio sujeto a través de la interacción con los demás. Esta teoría se basa en el proceso de asignación de símbolos con significados, el lenguaje hablado o escrito y el comportamiento en la interacción social (Martínez, 2006). En este sentido se resalta la importancia de las interacciones y transacciones que se establecen entre el niño y los elementos de su entorno, empezando por los padres y los iguales y continuando con los actores escolares que intervienen e influyen de manera significativa en su sistema de creencias y percepciones relacionadas con la alimentación. 
De acuerdo con estas ideas no se puede mirar el comportamiento del niño de forma aislada, como fruto exclusivo de su maduración, sino siempre en relación a las interacciones del ambiente en el que se desarrolla (Fuertes y Palmero, 1998).

Numerosos estudios sobre la obesidad infantil hacen énfasis en causalidades biológicas, psicológicas, económicas y culturales. Desde un enfoque social las posibilidades son diversas, ya que si consideramos que las personas en todo el desarrollo de su ciclo vital aprende valores, papeles y conductas de su cultura (Kail y Cavanaugh, 2008), el niño aprende a comer determinado tipo de alimentos, en ciertas cantidades, en un horario, con cierta frecuencia, bajo ciertas condiciones. Dicho aprendizaje en la primera etapa del ciclo vital recae en los padres y otros cuidadores primarios del grupo familiar, pero existen otros agentes sociales, instituciones y redes sociales que se van incorporando como fuerzas poderosas e influyentes en la generación de esas conductas, como la escuela, los amigos, los compañeros, la televisión, las redes sociales virtuales y otros, provocando actitudes y conductas de auto cuidado, pero también, otras que conducen a un deterioro en los estilos de vida.

El trabajo social como profesión práctica, requiere conocer la vida cotidiana de las personas, para identificar su objeto de intervención, y aunque las alternativas metodológicas son múltiples, uno de los mayores retos es el abordaje metodológico horizontal, participativo, holístico y adaptable a los sujetos; por lo cual, si el interés es conocer los aspectos relacionados con la prácticas alimentarias de los niños y niñas con sobrepeso y obesidad, una de las principales vías, es obtener el conocimiento de la realidad desde el sujeto que vive la problemática, siendo el principal protagonista de los procesos de cambio, y a partir de ello proponer con fundamento teórico y empírico, estrategias de intervención a través de mecanismos multidisciplinarios en distintos niveles y sectores sociales y participar en las estrategias de diseño, reformulación y evaluación de políticas sociales.

Considerando la magnitud de la problemàtica y a partir del reconocimiento de su multifactorialidad, la hipótesis de partida es que la obesidad infantil está asociada a diversos factores intrapersonales, familiares, escolares y a la cultura alimentaria regional. Por lo anterior, el propósito de esta comunicación es presentar las categorías identificadas en las prácticas alimentarias de los niños y niñas que presentan obesidad y cursan su educación primaria en escuelas públicas, así como algunos elementos que están relacionados con el aumento de peso y problemas que obstaculizan estilos de vida saludable con el fin de generar un mayor entendimiento de los contextos socioculturales y agentes implicados en las prácticas alimentarias. 


\section{Material y métodos}

El presente estudio inductivo-cualitativo intentó obtener evidencia científica de los entornos intrapersonales, familiares y escolares de los niños, vinculados con la alimentación en el contexto familiar y escolar. El muestreo fue intencional, es decir, se eligieron algunos casos con ciertas características de inclusión entre las que se encuentran que fueran alumnos regulares en escuelas públicas del Municipio de Monterrey y San Nicolás de los Garza Nuevo León en México, cursando el $4 .^{\circ}$ y $5 .^{\circ}$ grado en el periodo escolar del año 2011 y que presentaran obesidad. En el muestreo intencional de acuerdo a Hernández, Fernández y Baptista (2010), el investigador puede elegir ciertos casos, analizarlos y posteriormente compararlos con casos adicionales, homogéneos o heterogéneos. Este tipo de muestreo se caracteriza por un esfuerzo deliberado de obtener muestras «representativas» mediante la inclusión en la muestra de grupos típicos.

Con el fin de evitar la estigmatización, se entrevistó a un número similar de niños y niñas con peso normal, con sobrepeso y con obesidad, según la tabla que muestra «The International Obesity Task Force» (IOTF, 2002), se contó con información antropométrica recolectada por personal especializado en nutrición del Instituto Nacional de Salud Pública (INSP). De un total de 28 entrevistas, se eligieron 12 entrevistas que cumplían con el criterio de inclusión. Los padres firmaron el consentimiento de la participación de los niños seleccionados y una carta de asentimiento que ellos firmaron especificando el objetivo del estudio, los detalles de su participación, riesgos, beneficios y confidencialidad.

La principal técnica aplicada fue la entrevista cualitativa semi-estructurada a partir de la visualización de figuras de niños que mostraban un contorno diferente, como medio para provocar identificación y el discurso libre de cada uno de los niños y niñas. Los procedimientos de entrevista fueron validados y aprobados por los comités de ética e investigación del Instituto Nacional de Salud Pública en México (INSP). Las entrevistas fueron audio-grabadas y transcritas por personal calificado de la Facultad de Trabajo Social y Desarrollo Humano de la UANL. Con los discursos se estructuraron una serie de tópicos e ideas de las cuales emergieron categorías que fueron sistematizadas y ordenadas apoyadas por un procesador de textos denominado Ask Sam. La técnica de análisis de contenido fue de gran apoyo para comprender el mundo subjetivo de cada uno de los actores involucrados en el estudio (Creswell, 2009).

Estrategias tales como triangulación fueron utilizadas para complementar e incrementar el rigor del estudio. Para Padgett (2004) la triangulación puede 
ser: teórica, metodológica, de observadores y de datos; en este caso se utilizó la triangulación de datos. Para Hernández, Fernández y Baptista (2010), este tipo de triangulación permite la iluminación de la realidad desde diversos ángulos, para ello se utilizaron diferentes fuentes e instrumentos de recolección de datos en estancias prolongadas en campo, para hacer observaciones a la hora del descanso cuando los niños adquieren productos y/o consumen su almuerzo y a la hora de la salida de los niños que es ocasión propicia para comprar golosinas a vendedores ambulantes. Se realizaron tomas fotográficas y se escribieron notas de campo que pudieran corroborar los discursos de los niños y niñas del estudio, además se realizaron entrevistas cualitativas semiestructuradas a varios de los actores relacionados con el problema de la obesidad infantil (madres de los niños, maestros de grupo, profesores de educación física, directores, vendedores de cooperativas escolares, etc.) cuyos resultados se encuentran en otras publicaciones.

Tomando en cuenta la técnica de análisis de contenido, se efectuaron varios niveles de análisis de datos. Un primer análisis o evaluación de los hallazgos se realizó en un nivel individual, centrado en la persona, tratando de comprender el mundo subjetivo de cada uno de los participantes involucrados en el estudio, donde sus percepciones, actitudes, pensamientos y conductas, fueron aspectos relevantes a considerar; en un segundo nivel de análisis, se leyeron y re-leyeron los registros de las entrevistas, centrándose en la tarea de codificar, categorizar y recuperar segmentos de las entrevistas (Creswell, 2009).

\section{Resultados}

El niño y su entorno. Los ambientes de interacción y las categorías relacionadas con el problema de investigación

Con el análisis de los discursos se identificaron categorías relacionadas con la dieta habitual que consumen las niñas y los niños con obesidad en cada contexto, el tipo, cantidad y horario de alimentos, el consumo entre comidas y fuera de casa, la auto percepción de la figura, su autoestima, comportamientos ante los obstáculos al consumo de alimentos no saludables, tipo, tiempo y condiciones de actividad física, así como los problemas de interacción.

En el ámbito familiar destacan categorías como la relación entre aspectos socioeconómicos y la alimentación, la actividad laboral de los padres, la organización del tiempo y la alimentación, la forma de implicación del padre, la madre o/y otros cuidadores primarios en qué productos comprar, qué, cuánto 
y cómo comer, así como en la práctica de la actividad física de los niños y niñas.

Con relación a la escuela, el discurso se relaciona con la aplicación de la normativa oficial, los contenidos curriculares y competencias que se promueven en el ambiente escolar, la influencia de sus iguales, de los profesores y profesoras, los alimentos disponibles en la escuela, cooperativas escolares y negocios en la periferia de las escuelas, así como los tipos y características de la actividad física que se desarrolla en ese entorno.

Otros aspectos de interés encontrados en el estudio, son aquellos relacionados con la responsabilidad social de las empresas y la influencia de los medios masivos de comunicación en la modificación de los patrones alimentarios regionales.

A continuación se exponen algunas narraciones de la población en estudio, con el fin de caracterizar la situación que en materia de alimentación viven las niñas y los niños en los entornos familiar y escolar.

\section{Factores intrapersonales o del microsistema}

Autopercepción de los niños y niñas obesos

Para algunos niños y niñas la obesidad es algo que no atañe tanto a ellos «No, para mi edad y altura yo creo que sí estoy bien ... bueno ... me gustaría estar...no muy gordo ni muy flaco ... un poquito [más delgado], que no se me noten las lonjas» $(\mathrm{E} 6)^{3}$, pero en ocasiones la vida se complica y la obesidad no sólo altera la capacidad de movilidad y las interacciones sociales, sino que su autoestima se ve amenazada por actitudes de rechazo y burla que afectan la aceptación de sí mismo, la autoafirmación y la dignidad del niño:

«Cuando hablé en clase un niño dijo.. ya habló Santa Claus» (E2), «me dicen gordo unas veces» (E4) «Me hacen burla por el peso me dicen albóndiga o algo así ... no me gusta estar así, todos me dicen gordo, panzón»(E5) «te empiezan a decir... a discriminar... en mi casa mis primas me dicen cosas... En la escuela te dicen por ejemplo... ha visto la señora que le dicen «Big Mama» ... así me dicen» (E10).

\section{Factores familiares y escolares como mesosistema}

En el ambiente familiar, escolar y comunitario, los actores que se identifican en las narraciones de los niños y niñas, son los padres, hermanos, abuelas, personal de apoyo doméstico, administradores educativos, profesores de

3. Se identifica cada entrevistado con la letra $\mathrm{E}$ y un número progresivo. 
grupo y de educación física; participación de expendedores de comida en las escuelas, vendedores de golosinas en las periferias de los planteles, conductores y actores de radio y televisión. Todos ellos participan o contribuyen a diversas prácticas relacionadas con la alimentación y actividad física de los niños y niñas, favoreciendo y reproduciendo una cultura alimentaria regional. También hacen referencia a las tiendas de venta rápida de comestibles, así como expendio de alimentos procesados ubicados en su comunidad inmediata y en cada ruta de traslado a la escuela.

El discurso de los niños y niñas expresa la relación existente entre los aspectos socioeconómicos, actividad laboral de los padres, en especial de la madre, los cuidadores primarios sustitutos, las normas, disciplina e implicación de los padres en su alimentación.

Mi mamá tiene que trabajar....descansa nomás los lunes y no la hace (E1). Mi mamá trabaja de barrendera por una carretera, por la carretera «Libramiento» (lugar alejado del área metropolitana)... también trabajaba en «Oxxo».... (tienda de conveniencia) de aquí de abajo...Mi hermana prepara las comidas... sopas... a veces me hace carne... una señora nos vende 5 kilos de carne por $\$ 150$, es de res, de pollo, picadillo y deshebrada... es de ... (marca de baja calidad)... a veces me dan fruta picada en la mañana para que aguante hasta la tarde (E10).

Me dan poco dinero para gastar en la escuela....Porque no tienen ... (después de narrar que su alimentación es a base de tacos, expresa su gusto por algunas frutas y verduras pero reconoce que no las consume)...p porque mi mamá no tiene dinero pa' comprar... lo que cuesta ... a veces quiero sándwich... pero no tienen dinero pa' comprar pan ...Yo le diría a mi mamá que me traiga bastantes frutas y verduras ... o quiero que mi papá me inscriba en el americano de «Bronco» (Un Campo de futbol americano).. Sí, le estoy diciendo pero dice que cuando tenga dinero (E5).

La disponibilidad de comida, expresa que más allá de las carencias, las familias se esfuerzan por compensar con mayor cantidad, la menor calidad, lo que se refiere tanto a la comida como a la atención; las carencias económicas, las jornadas de trabajo de la madre y algunas situaciones de crisis familiar, en todos los casos provoca una reorganización de la función alimentaria con cuidadores sustitutos como abuelas, hermanas (os) que han abandonado la escuela, con situación de maternidad no planeada, lo que modifica las condiciones de alimentación del niño o de la niña. «A veces mi mamá no tiene tiempo para hacernos de desayunar y entonces comemos algo del súper... si no queremos algo nos venimos con nada (de comer) hasta la hora del recreo» (E7)... «no nos alcanza el tiempo (para desayunar antes de la escuela), porque llegamos tarde» (E6)... «Mi mamá trabaja y descansa nomás los lunes, ella no la hace (la comida), mi hermana es la que la hace» (E1) ... «Tengo obesidad porque 
a veces mi hermana como estaba chica no me podía dar de comer y mejor me compraba papitas y eso» (E10).

En las redes de apoyo encontramos frecuentemente personajes que ejercen involuntariamente la función de cuidador primario por crisis familiares o personales, como los hermanos o hermanas mayores: «La que se encarga de la comida es mi hermana de 16 años, (madre adolescente)» (E1)... «mi mamá, ya no trabaja, tiene una enfermedad que se cae el pelo (cáncer) y ella hace de comer y mi abuelita» (E4).

En la dinámica familiar nos encontramos a la obesidad infantil asociada a un problema relacionado con el sistema normativo y de valores familiares. El contexto social en el que funciona la familia actual hace que las decisiones sobre alimentación sean con frecuencia discutidas y negociadas por los niños y niñas, quienes tienen mucho poder en las decisiones del hogar por medio de la insistencia y la manipulación. Esta forma de funcionamiento está en parte determinada por el estatus laboral y las nuevas estructuras familiares, que hacen de la alimentación un acto rápido y centrado en la evitación de problemas «Compro lo que yo quiero....mis papás no saben lo que se vende ahí» (E2). «En casa... si me prohíben algo...me enojo y como lo que yo quiero» (E2). «Me prohíben las papitas con chile y me las como... todas» (E5).

El contexto de aprendizaje representado por la escuela, integra tanto la participación de los profesores que son los encargados de propiciar los aprendizajes significativos, como la gestión de los administradores educativos y el apoyo del personal técnico. Pero además aparecen actores como los profesores encargados de estimular la formación de hábitos de ejercitación física y otros, que son quienes atienden la preparación y expendio de alimentos que se adquieren dentro y fuera de las escuelas.

En general se advierte una casi nula oferta de alimentos saludables en las cooperativas escolares, la prohibición de alimentos no nutritivos se reduce a la disminución de salsas y comida picante, debido al alto índice de niños y niñas con algunos problemas gástricos detectados por los directivos y en ninguna de las escuelas del estudio se reduce la disponibilidad de alimentos fritos o con alto contenido calórico o de refrescos y jugos azucarados. Aunado a lo anterior el niño obeso llega al contexto escolar frecuentemente sin desayunar. Los niños y niñas entrevistados consumen con mucha frecuencia cereal azucarado con leche entera, compran de paso unas galletas con chocolate en las tiendas de conveniencia, llevan un lonche con alto contenido de grasa, tienen una cuenta abierta en la cooperativa para consumir lo que quieran o bien llevan dinero para comprar lo que deseen. «No como fruta en la escuela, porque aquí no venden, yo lo que más compro son las papitas de harina y coca 
(refresco de cola)» (E1). «Lo que más venden (en la escuela) es chatarra, la pizza....pero se les acaba rápido y también hay chili dogs...la mayoría (de lo que se vende) son dulces,... pero sí hay cosas nutritivas como jugos y aguas... si vendieran verduras yo no compraría» (E6).

Así queda demostrado que la escuela, tanto en las actividades realizadas en el aula como en las extraescolares, por su carácter de espacio educativo y por la cantidad de tiempo que permanecen en él los alumnos, ofrece innumerables oportunidades para formar hábitos alimentarios saludables y fomentar la práctica regular de actividad física y deporte, constituyendo, uno de los lugares más eficaces para modificar los estilos de vida de las y los niños en etapa escolar; sin embargo en el momento de la recolección de los datos, esto no sucedía.

\section{Los niños y niñas con obesidad y el patrón alimentario regional}

La variedad de alimentos en la dieta es necesaria para una buena nutrición y salud, pero también es un elemento de identidad cultural de la población. El Patrón de Conducta Alimentaria (PCA) está fuertemente asociado a factores culturales, nutricionales, socioeconómicos y demográficos; determinar el PCA es conocer lo que la población consume de manera cotidiana. México es un país rico en cultura alimentaria, por lo tanto el patrón de consumo puede variar de acuerdo a las regiones geográficas, culturales y económicas. ¿Qué alimentos consumen los niños y niñas obesos en las zonas urbanas del noreste de México? El siguiente es un caso ejemplificador de la ingesta cotidiana.

En la mañana...muy pocas veces, agarro un plátano o un paquete de galletas o me tomo unos vasos de agua... aquí (en la escuela) en la hora del recreo... como papitas, pizza y chili dog, o tacos de frijoles... o pocas veces mi mamá me manda lonche con .... mayonesa, queso y jamón, también hot dog ... y coca (refresco embotellado de cola) al salir de la escuela, una paleta helada o un Tampico (bebida a base de jugo de cítricos adicionados de azúcar)... taquitos de harina con frijoles... (en la comida) hamburguesas, tacos, tostadas con picadillo, pescado, pollo, a veces compramos pollo (asado)... arroz y elote ...en ocasiones ensalada; ... a veces me hacen sopa de brócoli... elote con mayonesa y un poco de chile en polvo... (con la comida]) tomo refresco y «Zuco» (polvo para preparar agua de sabores con azúcar) de limón...o melón ...Antes de que cene pos... muchas veces hago unas palomitas ...luego agarro unas galletas con un vaso de leche ... ceno ...la mayoría de veces tacos con frijoles y huevo con salchicha ....es lo que más ceno eh... todo, pero ¡ah! sí, también como muchas quesadillas ...con tortillas de harina. Cuando voy a casa de mi abuelita...nos hace bocoles... o sea gorditas... a mí me pone queso, aguacate y frijoles...y refresco ...o limonada (E6). 
En oposición al contenido del «plato del buen comer», los alimentos preferidos reiteradamente señalados en la mayoría de las entrevistas fueron: taquitos de tortilla de harina o de maíz con frijoles, o de chorizo con frijoles, de papa, barbacoa, de «trompo» (cerdo al pastor), de picadillo, frijolitos con huevo, huevo con chorizo, papas fritas, lonches con mayonesa, queso y jamón, cereales azucarados con leche entera, sopa de pasta (fideo y similares), arroz, hot dogs, chili dogs, hamburguesas, tostadas de frijoles, o con picadillo, pan, chocolate en caja ya preparado o en polvo, aguas azucaradas con sabor a fruta, refrescos embotellados de cola o de sabor a fruta, licuado de plátano con leche entera y frituras industrializadas.

En las narraciones se puede comprobar que los niños y niñas con obesidad no comen lo que quieren ni lo que saben que es nutritivo y saludable, sino más bien lo que pueden comprar o conseguir, y se enfrentan a dos situaciones: en primer lugar, pueden estar desnutridos debido a que no tienen los suficientes recursos para alimentarse y tienden a la obesidad porque los alimentos que consumen son de mala calidad, y por otro lado, las madres alimentan a sus hijos con productos industrializados baratos, puesto que los no procesados, como las frutas, verduras, carnes magras y lácteos, tienen mayor costo y están menos a su alcance, pero además implican un grado mayor de preparación (Cabello, Ávila y Garay, 2010). Entonces, la mayoría de los niños y niñas se alimentan de comida con altos contenidos en carbohidratos, grasas y azúcares que no les aportan saciedad, ni los nutrientes para tener una vida sana y peso adecuado.

Existe una disociación entre los conceptos que expresan los niños y niñas acerca de lo que debe ser una alimentación adecuada y sus competencias para una alimentación saludable, todos los niños y niñas con obesidad expresan de manera correcta cuales alimentos deben formar parte de su dieta, la importancia de beber agua, los beneficios del ejercicio, las causas de la obesidad y sus efectos en la salud; sin embargo, en sus discursos no se refleja la modificación de sus hábitos.

\section{Discusión}

Los resultados indican que los elementos vinculados al incremento del peso en las y los niños son diversos, pero principalmente están asociados a factores económicos, a la desorganización de la familia, a la alternancia de la función de diversos cuidadores primarios de los niños y las niñas y al desapego a las tareas que permiten el consumo de una alimentación sana, confirmando lo que señalan González, Muñoz, Naranjo y Nahar (2008) acerca del nivel de riesgo y el compromiso del grupo familiar en la problemática nutricional. 
Con relación al microsistema, a nivel personal los resultados muestran que la condición de obesidad genera problemas en la autoestima. Que hay una distancia entre el conocimiento que se tiene sobre una alimentación saludable y el desempeño de los niños, niñas y cuidadores primarios, manifestado en la elección de alimentos, las porciones y la frecuencia del comer, lo que plantea interrogantes de las competencias que en esta área han promovido los diferentes agentes que participan en la educación nutricional.

Los principales entornos de interacción social que forman el mesosistema donde se desarrolla la infancia, son el familiar y el escolar. Estos contextos tienen una fuerte influencia en las representaciones acerca del tamaño, contornos y forma del cuerpo, así como en la forma en el que no sólo cargan, sino viven el sobrepeso y la obesidad los niños y las niñas, afectando sus procesos de socialización y de educación. Los cuidadores primarios contribuyen significativamente al incremento del peso y posteriormente participan en los señalamientos del estatus de peso y de las diferencias con algunos procesos de exclusión que son aún más acentuados en el contexto escolar. Como señala Nuñez (2008) y se comprueba con los discursos, un niño con obesidad se siente diferente, ya que con frecuencia es objeto de burlas por sus grupos de amigos o compañeros. En la edad escolar, es frecuente encontrar este tipo de estigmatización de niños y niñas obesos por sus pares, lo que afecta la autoestima, promueve disconformidad con su propio cuerpo (Silvestri y Stavile, 2005) y afecta su socialización y su desarrollo.

La obesidad infantil de estos niños y niñas, se relaciona con dificultades en el funcionamiento de las familias y con problemas para ofrecer contención emocional y conductual. Un grupo familiar en el que se dedica tiempo y energía a cuidar la alimentación, seleccionar alimentos de calidad y diseñar menús variados genera un patrón muy diferente al de una familia en la que no se presta tanta atención a la alimentación, lo que se comprueba en numerosos estudios (Cabello, Ávila y Garay, 2010; Flodmark, Marcus y Britton, 2006; Restrepo y Maya, 2005; Núñez y Elizondo, 2004; Olivares, 2006).

Los discursos reafirman reiteradamente que la incorporación de la mujer a la vida laboral y los problemas en la organización familiar impactan de manera contundente en la modificación de hábitos alimentarios de la población infantil, y en las prácticas alimentarias (Romero, 2010).

Con relación al macrosistema se encontró que existen cambios en los patrones culturales, tanto objetivos como subjetivos que inciden en el incremento del peso, relacionados con la inversión y las estrategias de las empresas de la industria alimentaria, la penetración de hábitos alimentarios relacionados 
con la comida rápida y de alto contenido calórico, así como su accesibilidad. Es evidente que los patrones alimentarios regionales se han modificado.

Es interesante destacar el uso de la metodología cualitativa en el abordaje para la prevención y tratamiento de la problemática de la obesidad infantil. Se recomiendan estrategias de intervención basadas en las creencias, significados, ideas y pensamientos de las personas vinculadas con el problema, aplicar las premisas del interaccionismo simbólico que se basan primeramente en la creatividad del sujeto y en su capacidad de interactuación, para que las personas redefinan sus actos y establezcan previsiones que las lleven a nuevos tipos de comportamiento en una acción conjunta, en este caso en los contextos y con los sujetos del mesosistema. Los niños a partir de la reflexión moldeada por la interacción social, pueden aprehender nuevos significados y símbolos que les permitirá desenvolverse, actuar e interactuar en un contexto obesogéno, buscando la mejor opción para la adopción de un estilo de vida saludable.

Se propone una mayor atención a los significados y sentimientos, asociados a la adopción de estilos de vida saludable y en el principio de la acción electiva de las personas, acentuando el carácter activo de niños y niñas con obesidad, en correspondencia con lo señalado por Blumer (1969) que para poder interpretar y comprender los significados que los actores le dan al fenómeno, se debe ver la acción desde la posición del actor, y es en este sentido que se pretende incidir a través del trabajo social con grupos de niños, en la adopción de nuevos estilos de vida centrados en una activación física y en una alimentación saludable.

Desde el trabajo social, también es importante promover cambios en en el currículo académico, a través de una acción coordinada entre profesores, padres de familia y otros agentes sociales que participan en las prácticas alimentarias, a fin de desarrollar competencias que no sólo deberán estar comprendidas en asignaturas específicas (ciencias naturales, sociales y educación física), sino también formando parte de otros contenidos (educación para la ciudadanía, etc.). Esta medida requiere una acción normativa impulsada desde las Secretarías de Estado, hasta una concresión en cada contexto del desarrollo del niño, operacionalizada por cada niño y niña, cada miembro de su familia y los demás agentes sociales.

Una recomendación que surge del estudio versa en reforzar mediante talleres o actividades extraescolares, la iniciación de los niños/niñas y sus padres en el mundo de la cocina y la gastronomía, aprendiendo a comprar alimentos, prepararlos y cocinarlos. De este modo se estimularán comportamientos positivos que favorezcan la aceptación y desarrollo de unos hábitos alimentarios saludables, con una perspectiva de igualdad de género. Incluir 
en los cursos de formación del profesorado, materiales didácticos y orientaciones sobre alimentación y nutrición, y su incidencia sobre la salud, así como de la importancia de practicar regularmente actividad física.

Ante la situación que viven las madres de los niños y niñas que trabajan y que prefieren comer fuera de casa o solicitar comida del exterior, se sugiere estimular iniciativas a través de redes sociales y potenciar las acciones educativas en colaboración con otros sectores como las asociaciones de padres de familia, empresas de restauración colectiva, fundaciones, etc. para la preparación y venta de alimentos nutritivos y a bajo costo.

Se tiene la convicción de que los niños son capaces de reaccionar y de cambiar las conductas potencialmente perjudiciales para ellos, construyendo nuevas líneas de comportamiento y resignificando conceptos y acciones tendientes hacia un nuevo estilo de vida, aunque esto signifique luchar frente al poder que la cultura obesogénica y globalizada impone a sus miembros. El niño como sujeto de intervención, será el único capaz de transformar o descomponer los significados y de evaluar la toma de decisiones, es decir, los pros y contras de sus acciones (Del Fresno, 2011). Desde este enfoque, la intervención del trabajador social tiene por objeto lograr que en las diversas acciones de la vida cotidiana, el niño supere el vandalismo interaccional de una sociedad que promueve el consumo de productos obesogénicos y el sedentarismo, hacia un nuevo estilo de vida saludable.

\section{Conclusiones}

Al analizar el sentido y significado del discurso de los niños y niñas entrevistados, se pudo constatar que la obesidad infantil está íntimamente relacionada con la percepción que tiene el niño de sí mismo, con los patrones socioculturales alimentarios de los cuidadores primarios, con las condiciones socioeconómicas, la organización de la familia y el curriculum escolar formal y oculto entre otros, lo que incide en cambios en los patrones alimentarios y de actividad física.

Los niños y niñas que viven con obesidad luchan contra un estigma que afecta su autoestima y que a la vez los excluye de algunas actividades y procesos de socialización en los entornos familiar, escolar y social, por lo cual, desde el trabajo social es importante participar en el diseño y reformulación de política sociales y modelos de intervención que contribuyan a estilos de vida saludables. 


\section{Agradecimientos}

El artículo presenta algunos resultados del proyecto « Promoviendo Estilos de Vida Saludable de Alimentación y Actividad Física para la Prevención de la Obesidad Infantil en la zona Norte de México». Como parte de una estancia posdoctoral en el Instituto Nacional De Salud Pública (INSP), financiado por el Consejo Nacional de Ciencia y Tecnología (CONACyT) México.

\section{Bibliografía}

BAERT, P. (2001). La teoría social del siglo XX. Madrid: Alianza

BLUMER, H. (1969). Symbolic interactionism: Perspective and method. Nueva Jersey: PRENTICE HALL,

BRONFENBRENNER, U. (1979). The ecology of human development La ecología del desarrollo humano). Cambridge: Harvard University Press.

Cabello, M., Ávila, M., Garay, S. (2010). Percepción de las madres sobre una alimentación «saludable» y su relación con los hábitos alimenticios de los hijos. En M. Cabello y S.

GARAY (Coords.). Obesidad y prácticas alimentarias: impactos a la salud desde una visión multidisciplinaria. Monterrey: Universidad Autónoma de Nuevo León, 193-214

CResWell, J. W. (2009). Research design: Qualitative, Quantitative and mixed methods approaches. $3 \mathrm{ed}$. Los Ángeles USA: SAGE.

Encuesta nacional de salud y nutrición 2006. (2007). Resultados de Nutrición. Cuernavaca Morelos: Instituto Nacional de Salud Pública - Secretaría de Salud.

ENCUESTA NACIONAL DE SALUD Y NUTRICIÓN 2012. (2013). Resultados por entidad federativa. Nuevo León. Cuernavaca Morelos: Instituto Nacional de Salud Pública.

Flodmark, C., Marcus, C. Britton, M. (2006). Interventions to prevent obesity in children and adolescents: a systematic literature review. International Journal of Obesity, 30, 579-589.

Fuertes, J. y PAlmero, O. (1998). Intervención temprana. En M.A. Verdugo (Dir.). Personas con Discapacidad. Perspectivas psicopedagógicas y rehabilitadoras (2. ${ }^{a}$ Ed.) Madrid España: Siglo XXI.

González, W. Muñoz, N., Naranjo, C. \& Nahar, E. (2008) Abordaje social de la malnutrición. Vía para la construcción de capital humano y social. En Carballeda, A. (Coord.) Salud e intervención en lo social. Buenos Aires: Espacio Editorial.

Hernández, R., Fernández. C., BAPtista, P. (2010). Metodología de la investigación científica. (5a. ed.). México: Mc. Graw Hill. 
International Obesity Taskforce (IOTF) (2002). The Health of Children and Young People. Recuperado el 10 de enero de 2010, de http://www.iotf.org/ popout.asp?linkto=http://www.archive2.official-documents.co.uk/document/ deps/doh/survey02/hcyp/tables/hcypt159.htm

Kail, R.V., \& CaVanaugh, J.C. (2008). Desarrollo humano. Una perspectiva del ciclo vital. (3. ${ }^{a}$ ed.) México, D.F: CENGAGE Learning.

Martínez, M. (2006). Ciencia y arte en la metodología cualitativa. México: Editorial Trillas. 2. ${ }^{\mathrm{a}}$ edición.

NúÑEZ, H. Y ElizONDO, A. (2004). Elementos teóricos psico-socio-culturales por considerar en el diseño de una intervención educativa para combatir la obesidad escolar. Boletín inciensa, 16 (3), 2-7.

Olivares, S., Bustos, N., Moreno, X., Lera, L. y Cortez, S. (2006). Actitudes y prácticas sobre alimentación y actividad física en niños obesos y sus madres en Santiago, Chile. Revista Chilena de Nutrición, 33 (2), 170-179.

PADGETT D (2008). Qualitative methods in social work research. Challenges and rewards. USA: Sage Publications.

PAYNE, M. (1995). Teorías contemporáneas del trabajo social. Una introducción crítica. Barcelona: Paidós.

PÉREZ, G. (2004). Investigación cualitativa: retos e interrogantes I. Métodos. (4. ${ }^{\text {a }}$ ed.) Madrid España: Editorial la Muralla.

RESTREPO, S. y MAYA, M. (2005). La familia y su papel en la formación de los hábitos alimentarios en el escolar. Un acercamiento a la cotidianidad. Boletín de Antropología, 19 (36), 127-148.

Romero, J. (2010) Obesidad ¿Qué hacer? Políticas al vapor, problema de peso. http://politicasalvapor.com/system/files/Obesidad.pdf.

SALTIER, P. (2004). Llamado a entrar en acción plan contra la obesidad infantil. Boletín Práctica Médica Efectiva. http://www2.sdcounty.ca.gov/hhsa/ documents/CDHDChildhoodObesityActionPlanSpanVer.pdf

Silvestri E y Stavile E. (2005). Aspectos psicológicos de la obesidad. Córdova: Universidad Pavaloro. www.nutrinfo.com.ar/pagina/info/ob05-02.pdf

Villalba, C. (2004). La perspectiva ecológica en el trabajo social con infancia, adolescencia y familia. Portularia, 4, 287-298.

Wang, Y., Wu, Y., Wilson, R., Bleich, S., Cheskin, L., Weston, Ch., Showell, N., Fawolw, O., LaU, B., Segal, J. (2013). Childhood Obesity Prevention Programs. Comparative Effectiveness Review and Meta-Analysis, 115.

ANTECEDENTES DE DIFUSIÓN:

Un monográfico producto de un congreso de investigación cualitativa en salud. Con un enfoque teórico, categorización distinta de los datos, y propuesta desde otro modelo. 